\title{
La problématique de la distance dans la formation des médecins
}

\section{The distance issue in physicians' education}

\author{
Maria Grazia ALBANO ${ }^{1}$, Jean François d'IVERNOIS ${ }^{2}$ \\ 1 Centre de pédagogie des sciences de la santé, Faculté de médecine, Université de Foggia, Italie \\ 2 Laboratoire éducations et pratiques de santé (LEPS), Université Paris 13, France
}

Manuscrit reçu le 5 avril 2016; commentaires éditoriaux formulés aux auteurs le 6 septembre 2016 ; accepté pour publication le 15 octobre 2016.

\section{Mots-clés}

pédagogie médicale ; distance ; histoire de la médecine ; enseignement de la médecine

\section{Keywords}

medical education; distance; history of medicine; medical teaching

\begin{abstract}
Résumé - Problématique : Les étudiants médecine, aussi bien que les médecins, jugent sévèrement leur formation : trop théorique, trop loin de la vie réelle, trop loin des besoins des futurs professionnels et de la société. Dans cette réflexion sur la distance que l'enseignement de la médecine a prise vis à vis de son objet, nous avons considéré la nature multidimensionnelle de cet écart. Analyse : De façon critique, mais en reconnaissant les efforts déployés et les succès obtenus, nous avons analysé successivement la distance de la formation des médecins vis à vis du corps humain, la distance entre la théorie et la pratique, la distance par rapport aux besoins de santé de la population, au modèle bio psycho social, aux maladies chroniques et enfin, la distance entre l'enseignement et l'apprentissage. Conclusion : Cette réflexion, qui emprunte à l'histoire de la médecine et de l'éducation médicale, n'a pas d'autre prétention que de susciter le débat. Elle est également une invitation à mieux comprendre comment se créent et s'entretiennent des distances nuisibles à la compétence des futurs médecins, auxquelles souhaiterait remédier la pédagogie médicale.
\end{abstract}

\begin{abstract}
Introduction: Students and physicians consider graduate education too theoretical and too disconnected from real life, from the needs of future professionals and from society. Considering that medical teaching has distanced itself from its object we explored the multidimensional nature of the gap. Analysis: Maintaining a critical eye while recognizing the efforts made and the successes, we successively examined the distance of medical education with respect to the human body, the distance between theory and practice, the distance with respect to a population's health needs, the distance with respect to the biopsychosocial model, the distance with respect to chronic diseases, and lastly the distance between teaching and learning. Conclusion: This review, which borrows from the history of medicine and medical education, has no other claim but to foster a debate. It is also an invitation to better understand how distances detrimental to the competence of future physicians are built up and maintained. It is an issue medical education could resolve.
\end{abstract}




\section{Introduction}

C'est un reproche que l'on entend fréquemment adresser aux formations supérieures : leur distance vis à vis de la réalité. Les formations seraient trop théoriques, trop loin de la vraie vie, trop loin des besoins des futurs professionnels et de la société. Bien que depuis des siècles la formation des médecins soit à la fois théorique et pratique, ces reproches la concernent également. Ils proviennent aussi bien des praticiens qui accueillent les étudiants en stage que des étudiants en médecine eux-mêmes, qui, plusieurs années après la fin de leurs études, les jugent de façon sévère $^{[1]}$.

Nous avons voulu examiner de plus près cet argument de la distance que prendrait, à travers les époques, la formation médicale vis à vis de son objet.

Nous avons considéré le caractère multidimensionnel de cet écart et envisagé successivement la distance de l'enseignement médical vis à vis du corps humain, la distance entre théorie et pratique, la distance vis à vis des besoins de santé de la population, celles vis à vis du modèle bio psycho social ou vis à vis de la maladie chronique, et enfin la distance entre enseignement et apprentissage.

Il s'agit d'une réflexion qui emprunte à l'histoire de la médecine et à la pédagogie médicale mais n'a néanmoins d'autre prétention que de susciter le débat. D'autre part, les auteurs sont bien conscients qu'il existe des différences géographiques importantes dans l'approche et les modalités de la formation médicale, ce qui limite la portée de certains de nos propos. Néanmoins, cette tribune constitue une invitation à mieux comprendre comment se créent et s'entretiennent des distances nuisibles à la compétence des futurs médecins, auxquelles souhaiterait remédier la pédagogie médicale.

\section{Distance vis à vis du corps humain}

Historiquement, cette distance s'impose dès la création des premières écoles de médecine (Salerne, Bologne, Oxford, Montpellier, etc.). L'enseignement médical est éloigné du corps humain, non seulement parce que la dissection humaine est interdite, mais aussi parce qu'il est fondé sur les théories de Galien, un système de connaissances médicales basé originellement sur l'observation anatomique des animaux. Il faudra attendre quelques siècles avec les premières dissections anatomiques (tout d'abord clandestines) et surtout les théories de Harvey sur la circulation sanguine pour mettre un terme aux dangereux postulats de Galien ${ }^{[2]}$. Il s'en est suivi une vision plus réaliste et intégrée du corps dans la formation médicale.

On pourrait penser que ce progrès pédagogique s'accélèrerait aujourd'hui grâce aux procédés d'imagerie diagnostique et aux représentations anatomiques en 3D proposées aux étudiants sur les écrans de leur ordinateur. Mais ce rapprochement à la réalité du corps sain ou malade n'est que partiel. De l'avis de plusieurs enseignants cliniciens, les étudiants hospitaliers ont pris une distance inquiétante avec le corps de leurs patients. En raison d'une moindre attention de la formation à l'acquisition de compétences cliniques, ils semblent être moins habiles que leurs aînés à se servir de leurs sens pour repérer, grâce à un examen clinique attentif, le signe fin sur lequel ils pourraient fonder leur diagnostic. À peine le patient a-t-il fini d'évoquer son motif de consultation qu'il est trop souvent précipité dans un carrousel de coûteux examens biologiques ou d'imagerie : à eux de débrouiller les hypothèses diagnostiques !

La médecine semblerait donc, en raison des moyens technologiques d'investigation mis à sa disposition, perdre progressivement le contact avec le corps, celui que l'on inspecte, que l'on palpe, que l'on percute, que l'on ausculte ${ }^{[3]}$. Toutefois, des efforts importants ont été mis en œuvre dès les années 1980 dans la plupart des facultés de médecine pour faire acquérir aux étudiants ces compétences gestuelles, techniques et relationnelles dans des conditions non dommageables pour les patients, avec les «skills lab» qui sont devenus depuis des laboratoires de simulation.

\section{Distance dans la formation entre théorie et pratique}

Depuis le Moyen Age jusqu'au début du $18^{\text {ème }}$ siècle, l'enseignement de la médecine a été seulement 
théorique. La faculté interdisait aux étudiants de participer au traitement du patient. C'est seulement vers la fin du 17ème siècle que la chirurgie, discipline pratique par essence, fut élevée, à Paris, au rang de discipline académique ${ }^{[4]}$. Des siècles plus tard (et jusqu'à ce jour dans certains pays d'Europe), il aura été possible de devenir médecin malgré une expérience pratique des plus réduite. L'instauration, en France, dans les années 1970, d'un numerus clausus avait précisément pour but de limiter la production des médecins aux capacités de formation des hôpitaux. Cette officialisation de l'hôpital en tant «qu'école d'application » est directement dérivée des recommandations d'Abraham Flexner qui proposa en 1910 de rapprocher l'éducation, le soin et la recherche en les réunissant sous un même toit, celui d'un centre hospitalo-universitaire $^{[2]}$.

Toutefois, il aura fallu des décennies d'efforts de la part des «pédagogues médicaux » pour faire admettre aux instances dirigeantes des facultés de médecine que la formation pratique avait autant d'importance que la formation théorique et qu'il fallait par conséquent définir des objectifs de stage, varier ces derniers pour exposer les étudiants au plus grand nombre de situations professionnelles possibles, développer des stages de santé communautaire, accorder aux examens «cliniques » le même poids qu'aux épreuves théoriques. Aujourd'hui encore, le temps consacré dans certains pays par l'étudiant à sa « professionnalisation » est singulièrement court par rapport aux milliers d'heures qu'il doit passer à l'acquisition de sa culture scientifique. C'est majoritairement ce que les praticiens installés reprochent aux facultés qui les ont formés ${ }^{[1]}$. C'est aussi le constat dressé aujourd'hui pour certaines professions médicales, par exemple, les jeunes diplômés européens en chirurgie dentaire dont le manque de compétence pratique est souligné ${ }^{5]}$.

\section{Distance entre les programmes d'études, les besoins de santé de la population et le système de santé}

À l'Université, la médecine est enseignée par des spécialistes qui délivrent aux étudiants des connaissances sur les cas cliniques qu'ils traitent à l'hôpital ; leurs patients hospitalisés présentent presque toujours des pathologies d'un certain niveau de gravité ; elles sont souvent rares. Les programmes d'études ont été trop longtemps le reflet d'une pratique « hospitalo-centrée », tandis que les problèmes de santé les plus fréquents, ceux qui se rencontrent au niveau des soins de première ligne, n'étaient pas suffisamment pris en considération. Même si l'enseignement de la médecine générale, les stages obligatoires aux urgences et chez les médecins généralistes ont beaucoup amélioré ce problème, les habitudes prises par le jeune interne de prescrire larga manu un ensemble d'investigations (de routine, dans un contexte hospitalier) se retrouvent ensuite dans sa pratique en ville ou sur le territoire.

À un moment où les pays occidentaux connaissent de sérieuses difficultés financières et où les budgets consacrés à la santé sont en fort déficit, on ne peut plus accepter d'enseigner une médecine qui ne regarde pas au coût des examens ni des traitements prescrits. Les jeunes médecins doivent être, au contraire, formés au principe du « juste soin ».

Ainsi donc, l'hôpital a perdu progressivement son monopole de lieu de formation à l'exercice de la médecine. Dès la fin des années 70 ont été lancées à travers le monde des facultés expérimentales de médecine dont le curriculum était basé sur les problèmes de santé prioritaires de la communauté environnante (community oriented curiculum). Les plus célèbres d'entre elles étaient McMaster, Sherbrooke au Canada, Albuquerque aux Etats-Unis, Bersheeva en Israël, Maastricht aux Pays Bas. Ces facultés innovatrices se sont regroupées dans un réseau international très actif et influent d'écoles de médecine centrées sur les besoins de santé de la communauté : the Network for Community Oriented Educational Institutions for the Health Sciences ${ }^{[6]}$.

Plus récemment, l'Organisation mondiale de la santé (OMS) a souhaité rapprocher les facultés de médecine de leur environnement humain en mettant en avant leur « rôle social » ${ }^{[7]}$. Cette nouvelle mission des facultés, au-delà de leurs rôles traditionnels de production et de diffusion des savoirs, consiste à se mettre à l'écoute des besoins de santé régionaux, grâce en particulier à la recherche en santé publique, 
au développement de centres universitaires de santé communautaire et de programmes de promotion de la santé.

\section{Distance entre la formation basée sur le modèle bioclinique et la formation basée sur le modèle biopsychosocial}

Au cours des trente dernières années, il s'est produit un changement épistémologique important dans les programmes de formation médicale traditionnellement axés sur la physiopathologie, le diagnostic et le traitement des maladies. De fait, dans la plupart des facultés, on aurait plutôt tendance à enseigner comment traiter une pathologie que comment prendre soin d'une personne malade. Cependant, la communauté scientifique a progressivement accepté l'idée que le paradigme biomédical ne suffisait pas à expliquer la totalité de l'étiologie et de l'évolution des maladies. On croit donc, aujourd'hui, que la maladie ne résulte pas exclusivement d'un dérèglement de nature biologique mais d'un ensemble de déterminants de l'environnement psychologique, social, économique, écologique, dans lequel vit la personne.

Ce passage d'un modèle bioclinique dominant, sur lequel a été fondé pendant des siècles la philosophie et l'orientation des curricula, à l'actuel modèle, appelé «biopsychosocial » ${ }^{[8]}$, s'est traduit par des changements significatifs des objectifs et du contenu des programmes de formation. On note d'un côté, une forte augmentation des enseignements en santé publique, principalement l'épidémiologie et l' organisation des systèmes de santé. D'un autre côté, on a vu se renforcer en début de curriculum, à l'exemple des USA, des enseignements de sciences humaines (Humanities) : philosophie, histoire de la médecine, psychologie, sociologie de la santé, éthique, etc. Le but est ici de développer chez les étudiants une vision du patient qui ne se résume pas à une somme d'appareils sains ou défaillants, mais aussi de démontrer le caractère holistique de la médecine ${ }^{[9]}$. D'autre part, l'enseignement de la communication soignant-soigné a acquis une réelle importance grâce à l'organisation d'ateliers vidéo enregistrés impliquant des patients simulés. Aux États-Unis, au Canada, en Suisse, aux
Pays-Bas, des épreuves de simulation du dialogue soignant-soigné, mobilisant également des patients simulés, font désormais partie des examens nationaux requis pour obtenir le diplôme d'exercice. Récemment, les facultés de médecine de Montréal, de Laval, de Bobigny-Paris 13 ont fait appel à des patients pour expliquer aux étudiants "ce que cela signifie réellement d'être malade ${ }^{[10,11] . "}$

\section{Distance entre une médecine fondée sur les grands nombres et une médecine de la singularité}

\section{L'apparition de l'Evidence-Based Medicine} (EBM) ou médecine fondée sur les preuves a créé dans les années 90 une sorte de révolution copernicienne $^{[12]}$. Grâce aux analyses statistiques cumulant les résultats d'études internationales de même type sur le même sujet ou méta analyses il a été possible de faire émerger une vérité médicale à caractère universel, là où se combattaient auparavant des théories d'école. De là sont issues des recommandations (ou golden standards des pays anglo-saxons) publiés par les sociétés savantes ou par des organismes nationaux tels que la Haute autorité de santé, en France. Indiquant la norme du moment, la conduite à tenir pour la prise en charge d'un nombre important de maladies et de situations cliniques, ces recommandations ont été immédiatement intégrées dans le contenu des études médicales comme dans le programme du concours de l'internat. Elles sont devenues aujourd'hui la boussole exclusive des étudiants, au risque de leur faire négliger cet autre versant de la vérité médicale qu'est la singularité. En effet, de multiples maladies et syndromes rares échappent aux radars des statistiques, mais les malades présentent aussi fréquemment des spécificités cliniques qui les font exclure des cohortes. Il y a donc péril pédagogique à faire oublier au futur médecin l'unicité absolue du patient. C'est presque en réponse à une médecine fondée sur l'analyse de la multitude que s'est développé le courant de la médecine narrative, centré sur la singularité de la personne malade et de son histoire. 
La médecine narrative s'inscrit dans l'attention portée aujourd'hui aux aspects psychosociaux de la maladie $^{[13]}$. Il s'agit, par une analyse minutieuse du récit du patient, de recueillir les informations qui aident à comprendre sa problématique de santé, pour l'accompagner plus efficacement dans le processus d'acceptation de sa maladie chronique.

Même si tous les étudiants ne sont pas encore formés à cette méthodologie, ils peuvent y être sensibilisés dans les enseignements de spécialité en médecine générale et ceci peut les conduire ultérieurement à interroger différemment leurs patients. L'intérêt pédagogique est ici de tenter de rééquilibrer un enseignement médical trop imprégné de l'esprit et des conclusions de l'épidémiologie.

\section{Distance entre formation aux maladies aiguës et formation aux maladies chroniques}

Durant des siècles, les médecins ont été confrontés à des problèmes de santé aigus. Il en résulte qu'une partie très importante du curriculum est encore consacrée aux affections aiguës et aux moyens d'y faire face. Grâce à la vaccination, à l'hygiène, à la prévention, aux progrès de la thérapeutique, à l'élévation du niveau économique et culturel de la population, l'espérance de vie a augmenté considérablement au cours des dernières décennies. Le revers de cette victoire de la médecine est que les maladies chroniques sont devenues le problème de santé prioritaire et la principale cause de dépenses de santé dans les pays développés. Contrairement à la maladie aiguë qui nécessite une intervention rapide et efficace du médecin, la prise en charge de la maladie chronique implique de sa part un accompagnement prolongé du patient qui devient de facto, par la qualité de son observance au traitement, le principal décideur de l'évolution de sa maladie ${ }^{[14]}$. Le futur médecin devra donc apprendre à gérer le temps du soin autrement que dans l'immédiateté. Il apprendra à surmonter sa frustration vis à vis d'une observance à éclipses, souvent due à un déni, à une "fatigue" de se soigner au quotidien, à des difficultés psychologiques ou sociales, ou plus simplement à un manque de compréhension des mécanismes de la maladie et du traitement. Il devra, par exemple, comprendre l'intérêt du compromis thérapeutique, la portée d'une communication claire, le devoir d'éduquer.

Cet accompagnement requiert donc des compétences nouvelles de la part de l'étudiant, non encore suffisamment inscrites au programme des études. Il s'agit de conseiller le patient, de soutenir sa motivation à se soigner et surtout de le former à la gestion cognitive et psychologique de sa maladie : c'est l'éducation thérapeutique, qui constitue un véritable transfert de savoirs entre soignant et soigné ${ }^{[15]}$.

Mais cette éducation est difficile, car le patient est un apprenant particulier. La pédagogie du patient requiert donc une formation spécifique de l'étudiant en médecine ${ }^{[16]}$.

D'autre part, il est difficile d'assurer seul la prise en charge de patients chroniques, surtout si ceux-ci présentent simultanément plusieurs pathologies, comme c'est de plus en plus souvent le cas. C'est en fait à la multidisciplinarité et à la multiprofessionalité que doivent être initiés le plus tôt possible les étudiants, car c'est dans ce cadre qu'ils exerceront leur métier.

\section{Distance entre enseignement et apprentissage}

Une conviction assez courante dans l'enseignement supérieur est qu'il suffit d'enseigner pour faire apprendre. Pour cette raison, il a été communément admis pendant trop longtemps qu'une formation pédagogique des enseignants en médecine n'était pas nécessaire, alors qu'un des intérêts majeurs de cette formation est de faire comprendre, entre autres, la différence entre enseignement et apprentissage.

L'apprentissage de la médecine est particulièrement complexe, non seulement parce que le nombre de concepts à assimiler est très élevé (on parle, pour un curriculum complet, de l'équivalent de deux langues étrangères), mais aussi - et surtout - parce que les étudiants doivent intégrer mentalement ces concepts selon les registres distincts des sciences 
biologiques, cliniques, des sciences humaines et sociales et de la santé publique.

Tout problème de santé doit pouvoir être analysé à travers le prisme de ces différentes perspectives. Voilà pourquoi le modèle de curriculum proposé par Abraham Flexner, organisé en deux temps - enseignement des sciences fondamentales suivi de celui des sciences cliniques - n'est plus approprié aujourd'hui à la construction du métier de médecin, même s'il demeure encore la référence majoritaire dans le monde. De même, l'idée répandue selon laquelle la connaissance de la théorie doit nécessairement précéder l'application pratique a été contredite par des dizaines de recherches qui ont démontré que l'individu parvient mieux à saisir une règle générale à partir d'un exemple concret que le contraire. Ainsi, les avancées de la psychologie cognitive et des travaux issus la théorie de l'apprentissage social (social learning theory) ${ }^{[17]}$ nous ont permis de mieux comprendre les chemins qu'emprunte l'apprentissage. Les leçons qu'on peut en tirer vont complètement à l'encontre de la façon traditionnelle de faire acquérir des connaissances. Le cours magistral, même modernisé sous la forme enregistrée et illustrée du «podcast », reste un mode de transmission passif ; il peine à faire accrocher durablement des nouveaux concepts aux chaines de connaissances antérieures des étudiants.

Dès lors, une pédagogie qui associe la résolution de problèmes cliniques de patients à la compréhension des principes des sciences fondamentales, le tout dans le cadre de travaux de groupes impliquant la participation active des étudiants, a démontré son efficacité sur le plan de l'apprentissage ${ }^{[18]}$.

Elle a été adoptée dans les années 90 par plus de la moitié des facultés de médecine mondiales sous forme de curriculum organisé par problèmes, que ce soit le curriculum complet, un curriculum parallèle (parallel track) ou des portions d'années d'études, ou encore des blocs disciplinaires. Parallèlement, de nouvelles modalités d'évaluation ont vu le jour, comme l'Objective Structured Clinical Examination (OSCE) (examen clinique objectif structuré -ECOS), vérifiant dans un temps court un ensemble d'aptitudes et de techniques médicales, et le progress test ou test de progression, consistant en une série des questions à choix de réponse tirées d'une base de connaissances essentielles ; appliquée chaque année aux étudiants elle se traduit par une exigence de performance croissante $^{[19]}$.

Aujourd'hui, l'investissement pédagogique des facultés de médecine est mis sur l'e-learning (apprentissage à distance en ligne), compte tenu des fantastiques possibilités offertes par l'internet : qualité du contenu scientifique, richesse, diversité des illustrations, possibilités d'auto évaluation et même de dialogue médiatisé avec les enseignants. Si cette approche prend en compte les rythmes individuels d'apprentissage des étudiants, elle n'est pas, a priori, orientée vers le développement de leur capacité à travailler en équipe et ne peut donc constituer la seule méthode pédagogique utilisée en médecine.

Les centres ou laboratoires de simulation constituent une autre avancée de la pédagogie médicale. Inspirés de l'expérience déjà longue des facultés de médecine américaines, ils constituent le moyen de faire acquérir aux étudiants des habiletés techniques, manuelles ou relationnelles, dans un contexte non nocif pour le patient. Ceci est particulièrement important quand on sait que la compétence en médecine résulte de la cumulation et d'une intégration de savoirs, de savoirs faire techniques et de savoir être.

\section{Conclusion}

La réflexion critique menée jusqu'ici doit certainement être nuancée, d'autant plus qu'elle s'inscrit dans les limites d'un texte d'opinion. En fait, aucune formation professionnelle n'est tout à fait pertinente ; celle des médecins n'échappe pas à ce constat. La médecine, comme les autres disciplines scientifiques (et peut-être plus que d'autres), a connu au cours des 50 dernières années une augmentation exponentielle des connaissances, qualifiée par certains « d'explosion des connaissances ». À titre d'exemple, la base de données Medline (http://www.nlm.nih.gov/services/usemedline.html) analyse chaque année plus de 5600 revues médicales. Chaque connaissance est constamment remise en question. Face à ce phénomène, la tentation des enseignants chercheurs a été de se réfugier dans les frontières de leurs disciplines respectives, mais il est presque impossible aujourd'hui 
de tout savoir, même dans les limites étroites d'une sous spécialisation. Le résultat en est un certain « égoïsme disciplinaire » qui nuit à un programme de formation qu'on voudrait holistique. Chaque spécialité tente de défendre son arpent de connaissances mais à la fin, le savoir utile que tout futur médecin devrait posséder ne peut pas être la somme de ce que tous les différents spécialistes aimeraient qu'il sache.

Cependant, des progrès significatifs ont été accomplis au cours des trente dernières années dans la réflexion sur les finalités et le contenu des études médicales ${ }^{[20]}$, à tel effet qu'on peut considérer aujourd'hui que plusieurs importantes « distances » que nous avons décrites ont été réduites par de très nombreuses facultés de médecine à travers le monde. Des organismes comme l'American Association of American Medical Colleges (AAMC) dans son rapport : "Rapport GPEP : Médecins pour le $21^{\text {ème }}$ siècle » ${ }^{[21]}$, ont défini les compétences attendues des futurs médecins. De nombreuses facultés de médecine à travers le monde ont adopté un modèle dit « outcome based curriculum » (curriculum fondé sur des buts), qui décrit avec précision les objectifs du programme ${ }^{[22]}$. À la Faculté de Maastricht, les enseignants ont réussi à définir le socle indispensable de connaissances (core curriculum) que tous les étudiants doivent maitriser en fin d'études pour pouvoir exercer la médecine ${ }^{[23]}$.

Mais ce ne sont pas seulement les connaissances qui ont changé, ce sont aussi les compétences médicales, en raison des innovations technologiques, des contraintes économiques, juridiques et éthiques de l'exercice, des transformations du système de santé, des différents besoins de la population, des nouveaux rôles respectifs des patients et des médecins. L'approche par compétences (APC) s'est progressivement imposée comme le moyen de rendre le curriculum plus pertinent ${ }^{[24]}$.

Former des professionnels capables de répondre à toutes ces exigences, nécessite de réduire - voire d'éliminer- les distances que nous avons décrites. Ce défi n'est possible que si l'architecture des programmes d'études en médecine résulte d'une coconstruction par les différents acteurs : non seulement les médecins, enseignants - chercheurs universitaires, aidés par des spécialistes en pédagogie de la santé, mais aussi les responsables des politiques de santé, des patients, des représentants de communauté ${ }^{25,26]}$.

Mais ce projet est encore seulement au stade de la réflexion.

\section{Contributions}

Maria Grazia Albano et Jean François d'Ivernois ont co-rédigé solidairement le manuscrit

\section{Déclarations d'intérêts}

Les auteurs ne déclarent aucun conflit d'intérêts en lien avec le contenu de cet article

\section{Approbation éthique}

Sans objet

\section{Remerciements}

Les auteurs souhaitent remercier Vincent de Andrade (LEPS) pour son aide documentaire.

\section{Références}

1. Brun D, Hassid S, d'Ivernois J.-F. « Enquête rétrospective auprès de 4 promotions d'étudiants en médecine, 8 à 11 ans après la fin de leur formation médicale ». Presse Med 1999;28:1121-7

2. Calman KC. Medical education: past, present, and future: handing on learning. Edinburgh. New York: Churchill Livingstone, 2007.

3. Masquelet AC, Doron CO, Centre Georges Canguilhem. Le corps relégué. Paris: Presses universitaires de France, 2007.

4. Millepierres-F. La vie quotidienne des médecins au temps de Molière. Paris: Le Livre de Poche, 1990.

5. Brafman N. Des dentistes diplômés sans soigner de patients. Le Monde, vendredi 19 août 2016, p. 8. 
[On-line]. Disponible sur : http://www.lemonde.fr/ societe/article/2016/08/17/en-europe-10-des-diplomesen-chirurgie-dentaire-n-ont-jamais-soigne-un-patient_ 4983724_3224.html

6. Schmidt HG, Neufeld VR, Nooman ZM, Ogunbode T. Network of community-oriented educational institutions for the health sciences. Acad Med 1991;66: 259-63.

7. Boelen C. Consensus Mondial sur la Responsabilité Sociale des Facultés de Médecine. Santé Publique 2011;23:247-50.

8. Engel GL. The clinical application of the biopsychosocial model. Am J Psychiatry 1980;137:535-44.

9. Fantini B, Lambrichs LL (dir.) Histoire de la pensée médicale contemporaine: évolutions, découvertes, controverses. Tome 4. Paris: Éd. du Seuil, 2014.

10. Flora L. Le patient formateur auprès des étudiants en médecine: De l'approche historique, la contextualisation, à l'intervention socio-éducative [Mémoire de recherche de Sciences de l'Education]. Saint-Denis: Université Paris 8, 2008.

11. Gross O, Ruelle Y, Gagnayre R, Les patients enseignants, une révolution dans la formation des médecins. Le Monde festival. 12 septembre 2016 [On-line]. Disponible sur : http://www.lemonde.fr/ festival/article/2016/09/12/les-patients-enseignants-une-revolution-dans-la-formation-desmedecins_4996489_4415198.html\#RC34O0k7BOI EcLeJ.99

12. Sackett DL, Rosenberg WM, Gray JA, Haynes RB, Richardson WS. Evidence based medicine: what it is and what it isn't. BMJ 1996;312:71-2.

13. Charon R. Narrative Medicine: Honoring the Stories of Illness. 1st ed. New York: Oxford University Press, 2008.

14. Lacroix A, Assal J-P. L'éducation thérapeutique des patients : Accompagner les patients avec une maladie chronique : nouvelles approches. Paris : Maloine, 2011.

15. World Health Organization. Therapeutic Patient Education : continuing education programmes for health care providers in the field of prevention of chronic diseases. Copenhagen: World Health Organization, regional office for Europe, 1998.

16. Ivernois (d') J.-F, Gagnayre R. Apprendre à éduquer le patient, approche pédagogique : l'école de Bobigny. 5ème ed. Paris : Maloine, 2016
17. Bandura A. Social learning theory. Englewood Cliffs (NJ) : Prentice -Hall, 1977

18. Norman GR, Schmidt HG. Effectiveness of problembased learning curricula: theory, practice and paper darts. Med Educ 2000;34:721-8.

19. Albano MG, Cavallo F, Hoogenboom R, Magni F, Majoor G, Manenti F et al. An international comparison of knowledge levels of medical students: the Maastricht Progress Test. Med Educ 1996;30: 239-45.

20. Walton H. A global strategy for medical education: partners in reform. Med Educ 1993;27:394-8.

21. Association of American Medical Colleges. Physicians for the Twenty-First Century. The GPEP Report: Report of the Panel on the General Professional Education of the Physician and College Preparation for Medicine. Washington (DC) : Association of American Medical Colleges, 1984.

22. Harden RM. AMEE Guide No. 14: Outcome-based education: Part 1-An introduction to outcome-based education. Med Teach 1999;21:7-14.

23. Universiteit Maastricht. New Maastricht curriculum. 2001 [On-line]. Disponible sur : http://www.fmv.ulg. ac.be/upload/docs/application/pdf/2010-03/crmaastricht.pdf

24. Parent F, Jouquan J. Comment élaborer et analyser un référentiel de compétences en santé ? Une clarification conceptuelle et méthodologique de l'approche par compétences. Louvain-la-Neuve : De Boeck Supérieur, 2015.

25. de van Niekerk JP, Christensen L, Karle H, Lindgren S, Nystrup J. WFME Global Standards in Medical Education: status and perspectives following the 2003 WFME World Conference. Med Educ 2003;37: 1050-4.

26. Albano MG, d'Ivernois JF. Formazione iniziale in medicina e nelle professioni sanitarie. In : F. Calamo Specchia (a cura di). Manuale critico di Sanità Pubblica. Santarcangelo di Romagna : Maggioli ed., 2015

Correspondance et offprints : Jean François d'Ivernois, Laboratoire éducations et pratiques de santé, Université Paris 13, 74 rue Marcel Cachin, 93017 Bobigny Cedex, France

Mailto : ivernois.jf@orange.fr et Maria Grazia Albano, Faculté de médecine, Viale L. Pinto 1, 71121 Foggia, Italie.

Mailto : mariagrazia.albano@unifg.it 